\title{
Development of Spinning Equipment for Biconical Shaped Charge Liner \\ Yu Yang
}

\author{
Changchun University of Science and Technology,Changchun, Jilin province, China \\ yangyu1983@sina.com
}

\begin{abstract}
Key words:Biconical Shaped Charge Liner; Spinning Force Algorithm; Dimensional Precision Abstract.The biconical shaped charge liner serves as the main part to constitute the armour-piercing projectile and its dimensional precision is mainly affected by the spinning force. Excessive spinning force will cause the increase in feed amount of spinning roller and then raise the surface roughness of the shaped charge liner; otherwise, the insufficient spinning force will give rise to the decrease in feed amount of spinning roller and then enlarge the dimensional precision of diameter of the shaped charge liner. During the development of the equipment, the mathematical modeling is established for the accurate calculation of the spinning force in stead of the fixed spinning force traditionally utilized. It is proved by the spinning process experiment of the biconical shaped charge liner that this equipment can meet the required quality for spinning products and offer the dimensional precision obviously improved when compared with the shaped charge liners produced by traditional methods.
\end{abstract}

\section{Introduction}

The biconical shaped charge liner serves as the main part to constitute the armour-piercing projectile and its molding quality is mainly influenced by the dimensional precision of fixed points; the precision of the two points which are $7 \mathrm{~mm}$ and $32 \mathrm{~mm}$ far from the root is required to be $1.67 \pm 0.05 \mathrm{~mm}$ and $1.5 \pm 0.05 \mathrm{~mm}$ respectively. With a view to meeting the design requirement, this paper has made a study on the development of spinning equipment for the biconical shaped charge liner and established mathematical modeling for the accurate calculation of the spinning force in stead of the fixed spinning force traditionally applied. It is proved by the spinning process experiment that this equipment can produce the biconical shaped charge liners with a dimensional precision higher than that made by the equipment of the same kind and meet the required quality for spinning products.

\section{Composition of Equipment and Principles of Processing}

The spinning equipment of biconical shaped charge liners mainly comprise spindle box, lathe bed, cross-shaped sliding table, hydraulic system and electric control system ${ }^{[1]}$.

Spindle box: Its motor drives the spindle box to rotate through the narrow $\mathrm{V}$ shaped belt and the mandrel is installed on the spindle box.

Lathe bed: That the structure of lathe bed comprises the main lathe bed and side lathe bed, which are connected by bolts and leveled by adjusting screws.

Cross-shaped sliding table: It is mainly used for the horizontal and longitudinal feed. The front of sliding table is installed with spinning rollers; and the entrance angle of the spinning rollers is $20^{\circ}$.

Hydraulic system and electric control system: the hydraulic system mainly refers to the hydraulic station and primarily functions to offer power to the cross-shaped sliding table. The Siemens control system is adopted as the electric control system, mainly comprising two parts: control console and electric control cabinet.

The working principles of biconical shaped charge liner for metal spinning: install the plate blank onto the mandrel and make the workblank rotate together with the mandrel during spinning process; utilize the spinning roller to carry out longitudinal and horizontal feed and make it rotate relative to the workblank. The feed line of spinning roller is controlled by the playback type of numerical control system. The workblank will be pressed and produce the continuous local plastic deformation in order to obtain the biconical shaped charge liner. 


\section{Algorithm of Spinning Force and Power}

The following assumptions are required to be made before calculating the spinning forces of the biconical shaped charge liner:

1)The materials subject to deformation meet the required homogeneity and isotropy.

2)No negative volume is produced before and after the deformation of materials.

3)Neglecting the force of friction produced between the spinning roller and workblank.

4)The spinning roller and mandrel are defined to be rigid.

5)The materials within the deformation zone are regarded in a plane deformation state.

6)The deformation is defined to be the pure shear typed one ${ }^{[2-5]}$.

The strain velocity component of element includes the strain velocity of extension expressed by $\varepsilon_{R R}, \varepsilon_{\theta \theta}$ and $\varepsilon_{Z Z}$ and the strain velocity of shearing expressed by $\gamma_{R Z}, \gamma_{R \theta}$ and $\gamma_{R \theta}$, then:

$$
\begin{aligned}
& \left\{\begin{array}{l}
\varepsilon_{R R}=\frac{\partial v_{R}}{\partial R} \\
\varepsilon_{\theta \theta}=\frac{v_{R}}{R}+\frac{\partial v_{\theta}}{R \partial \theta} \\
\varepsilon_{Z Z}=\frac{\partial v_{\theta}}{\partial Z}
\end{array}\right. \\
& \left\{\begin{array}{l}
\gamma_{R \theta}=\frac{1}{2}\left(\frac{\partial v_{R}}{R \partial_{\theta}}+\frac{\partial v_{\theta}}{\partial R}-\frac{v_{\theta}}{R}\right) \\
\gamma_{R Z}=\frac{1}{2}\left(\frac{\partial v_{R}}{\partial Z}+\frac{\partial v_{Z}}{\partial R}\right) \\
\gamma_{Z \theta}=\frac{1}{2}\left(\frac{\partial v_{\theta}}{\partial Z}+\frac{\partial v_{Z}}{\partial \theta}\right)
\end{array}\right.
\end{aligned}
$$

In the equations above, $v_{R}, v_{\theta}$ and $v_{Z}$ represent the deformation velocity component of the element selected. As the radial position of the element remains unchanged in the process of deformation, then:

$$
v_{R}=\frac{d R}{d T}
$$

In the equation, $T$ represents Time.

In the process of deformation, the angular spin velocity is a constant and hence the tangential velocity component of the element for deformation is expressed as follows:

$$
v_{\theta}=2 \pi R n
$$

The material in contact with the spinning rollers in the process of deformation will be shaped to the form of spinning roller; hence, the axial displacement of the element, $Z$ can be expressed as follows:

$$
\left\{\begin{array}{l}
Z=f\left(R, \theta, n_{\gamma}\right) \\
d Z=\frac{\partial Z}{\partial R} d R+\frac{\partial Z}{\partial \theta} d \theta+\frac{\partial Z}{\partial n_{\gamma}} d n_{\gamma}
\end{array}\right.
$$

In the equations above, $n_{\gamma}$ represents the number of revolutions of the workblank within the instant of time $T-T_{0}$.

$\left\{\begin{array}{l}n_{\gamma}=n\left(T-T_{0}\right) \\ \frac{d n_{\gamma}}{d T}=n\end{array}\right.$

The axial velocity component of the element for deformation is expressed as follows:

$v_{z}=\frac{d Z}{d T}=\frac{\partial Z}{\partial R} \cdot \frac{d R}{d T}+\frac{\partial Z}{\partial \theta} \cdot \frac{d \theta}{d T}+\frac{\partial Z}{\partial n_{\gamma}} \cdot \frac{d n_{\gamma}}{d T}=n\left(2 \pi \frac{\partial Z}{\partial \theta}+\frac{\partial Z}{\partial n_{\gamma}}\right)$

The radial variable velocity component is expressed as follows:

$\gamma_{R Z}=\frac{1}{2} \cdot \frac{\partial v_{Z}}{\partial \theta}$

The tangential variable velocity component is expressed as follows: 


$$
\left.\gamma_{\theta Z}=\frac{1}{2 R} \cdot \frac{\partial v_{Z}}{\partial \theta}\right)
$$

The relation between the stress and strain velocity is expressed as follows:

$$
\varepsilon_{i j}=\mu S_{i j}
$$

In the equation, $\varepsilon_{i j}$ represents stress; $\mu$ represents the function of plastic modulus of material; $S_{i j}$ represents the strain velocity.

The second invariant of the deviator of strain velocity, $I$ is expressed as follows:

$$
I=\frac{1}{2} \sum\left(\varepsilon_{i j}\right)^{2}+\sum\left(\frac{\gamma_{i j}}{2}\right)^{2}
$$

The second invariant of the deviator of the stress, $J$ is expressed as follows:

$$
J=\frac{1}{2} \sum S_{i j}^{2}+\sum \tau_{i j}^{2}
$$

Its yield condition is expressed as follows:

$$
J=\tau_{0}^{2}
$$

In the equation, $\tau_{0}$ represents the yield limit of materials during pure shearing ${ }^{[6]}$.

$$
\begin{aligned}
& \tau_{0}=\frac{\sigma_{0.2}}{\sqrt{3}} \\
& \mu=\frac{\sqrt{I}}{\tau_{0}}=\frac{\sqrt{\left(\frac{1}{2} \gamma_{R Z}\right)^{2}+\left(\frac{1}{2} \gamma_{\theta Z}\right)^{2}}}{\tau_{0}}
\end{aligned}
$$

The principal component of the stress field is expressed as follows:

$$
\begin{gathered}
S_{R Z}=\tau_{R Z}=\frac{2 \tau_{0} \gamma_{R Z}}{\sqrt{\gamma_{R Z}^{2}} \delta \gamma_{\theta Z}^{2}}=\frac{2 \sigma_{0.2}}{\sqrt{3}} \cdot \frac{\gamma_{R Z}}{\sqrt{\gamma_{R Z}^{2}}} \\
S_{0.2}=\tau_{\theta Z}=\frac{2 \tau_{0 Z}^{2} \gamma_{\theta Z}}{\sqrt{\gamma_{R Z}^{2} \delta \gamma_{\theta Z}^{2}}}=\frac{2 \sigma_{0.2}}{\sqrt{3}} \cdot \frac{\gamma_{\theta Z}}{\sqrt{\gamma_{R Z}^{2}+\gamma_{\theta Z}^{2}}}
\end{gathered}
$$

Based on the assumptions that no negative volume occur in the process of deformation, the deformation power of unit volume, $w$ is the product of the deviator of stress and the deviator of the stress velocity:

$$
w=S_{i j} \varepsilon_{i j}=\tau_{R Z} \gamma_{R Z}+\tau_{\theta Z} \gamma_{\theta Z}=\frac{\sigma_{0.2}}{\sqrt{3}} \sqrt{\left(\frac{\partial v_{Z}}{\partial R}\right)^{2}+\frac{1}{R}\left(\frac{\partial v_{Z}}{\partial \theta}\right)^{2}}
$$

The power consumed in the whole deformation zone is expressed as follows:

$$
W=\int w d V_{o l}=\frac{\sigma_{02}}{\sqrt{3}} t_{0} \int_{\theta} \int_{R} R \sqrt{\left(\frac{\partial v_{Z}}{\partial R}\right)^{2}+\left(\frac{\partial v}{R \partial \theta}\right)^{2}} d \theta d R
$$

The spinning forces in tangential, radial and axial directions are expressed as follows respectively:

$$
\begin{aligned}
P_{t} & =\frac{W}{2 \pi R n} \\
P_{r} & =P_{t} \frac{(1-\sin \alpha) R_{0} \theta}{f \cos ^{2} \alpha} \\
P_{Z} & =P_{t} \frac{R_{0} \theta}{f \cos \alpha}
\end{aligned}
$$

In the equations above, $R_{0}$ represents the induction radius. 


\section{Spinning Process Experiment}

After the spinning equipment for the biconical shaped charge liner is installed and debugged, this algorithm is utilized to load the spinning force and conduct the spinning process experiment. The experiment parameters involved are listed in Table 1:

Table 1 Experimental parameters

\begin{tabular}{|l|l|l|}
\hline \multicolumn{2}{|l|}{ Parameters } & Numerical \\
\hline \multirow{3}{*}{ Blank } & Width & $70 \mathrm{~mm}$ \\
\cline { 2 - 3 } & Thickness & $4.4 \mathrm{~mm}$ \\
\cline { 2 - 3 } & Length & $70 \mathrm{~mm}$ \\
\cline { 2 - 3 } & Material & $\mathrm{Cu}$ \\
\hline \multirow{3}{*}{ Rotary wheel } & Diameter & $200 \mathrm{~mm}$ \\
\cline { 2 - 3 } & Fillet radius & $10 \mathrm{~mm}$ \\
\cline { 2 - 3 } & Installation angle & $20^{\circ}$ \\
\hline \multirow{2}{*}{ Core mold } & Max diameter & $60 \mathrm{~mm}$ \\
\cline { 2 - 3 } & Length & $125 \mathrm{~mm}$ \\
\hline \multirow{2}{*}{ Feed } & Spindle speed & $50 \mathrm{r} / \mathrm{min}$ \\
\cline { 2 - 3 } & Feed speed & $3 \mathrm{~mm} / \mathrm{r}$ \\
\hline
\end{tabular}

The key for the molding quality of the biconical shaped charge liner lies in the valve of wall thickness of the points which are $7 \mathrm{~mm}$ and $32 \mathrm{~mm}$ far from the root and the tolerance is required to be within 0.05 . It is proved by the spinning process experiment that the biconical shaped charge liners spun are fully qualified. The results of experiment are shown in Table 2.

Table 2 Experimental results

\begin{tabular}{|l|l|l|}
\hline & $7 \mathrm{~mm}$ Position & $32 \mathrm{~mm}$ Position \\
\hline Requirement & $1.67 \pm 0.05 \mathrm{~mm}$ & $1.5 \pm 0.05 \mathrm{~mm}$ \\
\hline First text & $1.70 \mathrm{~mm}$ & $1.51 \mathrm{~mm}$ \\
\hline Second text & $1.69 \mathrm{~mm}$ & $1.51 \mathrm{~mm}$ \\
\hline Third test & $1.70 \mathrm{~mm}$ & $1.53 \mathrm{~mm}$ \\
\hline Fourth test & $1.68 \mathrm{~mm}$ & $1.52 \mathrm{~mm}$ \\
\hline Fifth test & $1.68 \mathrm{~mm}$ & $1.52 \mathrm{~mm}$ \\
\hline Fixed value test & $1.72 \mathrm{~mm}$ & $1.55 \mathrm{~mm}$ \\
\hline
\end{tabular}

\section{Conclusion}

The results of the spinning process experiment made for the molding of biconical shaped charge liner show that compared with the traditionally utilized algorithm, this algorithm can effectively improve the dimensional precision of the biconical shaped charge liner and allows the equipment to realize more rapid determination of process parameter than the equipment of the same kind and shorten the time and frequency of spinning process experiment required to determine the optimal process parameters. 


\section{References}

[1] Zhao Yunhao, Li Yanli. Technoligy and application of spinning. Beijin: Machinery Industry Press, (2008)

[2] Matsunok. Recent Research and Development in Metal Forming in Japan, J, Mater, Process, Technol, Vol.2(1997),p.1-3

[3] Anon. Metal spinning in the auto motive industry,J, Sheet Metal Industries, Vol.5(1995),p.13-18

[4] Xue Kemin, Wang Zhen, Lu Yan. Elasto-plastic FEM Analysis and Experimental Study of

Diametral Growth in Tube Spinning, J, Mater, Process, Technol,Vol.172-175(1997),p.69

[5] WANG Z T, XIE S S, JIN Q J. Elasto-plastic finite element analysis of hydrostatic extrusion with various mathematically contoured dies,J, Proceedings of the 24th International Machine Tool Design and Research Conference, eds, Davies BJ, Vol.52(1983),p.51-58

[6] HAYAMA, Hiroaki KUDO. Experimental Study of Tube Spinning,J, Bulletion of JSME. Vol.769-775(1979,p.167 